\title{
Quantos Dias, Quais Horários e Quantas Medidas por Dia Devem Ser Recomendadas na Monitorização Residencial da Pressão Arterial?
}

\author{
How Many Days, Which Period of the Day and How Many Measurements per \\ Day are Recommended in Home Blood Pressure Monitoring?
}

Audes Diógenes de Magalhães Feitosa, Marco Antônio Mota Gomes, Décio Mion Júnior

Departamento de Hipertensão - MAPA e MRPA do RealCor - Real Hospital Português de Beneficência em Pernambuco, Clinicor - Clínica de Cardiologia de Maceió, Universidade de São Paulo - Pernambuco, PE - Maceió, AL - São Paulo, SP

Em 2001, foram publicadas as primeiras diretrizes brasileiras para o uso da monitorização residencial da pressão arterial (MRPA). Essas diretrizes relatavam que apesar dos vários protocolos de MRPA utilizados na época, não havia uma padronização. Então foram recomendadas ao menos duas medidas pela manhã, antes das medicações e do desjejum, duas à noite, antes do jantar ou três horas depois, para evitar a redução pós-prandial da pressão arterial (PA). Isso por, no mínimo, três dias consecutivos de atividades habituais, e desprezando-se as medidas do primeiro dia ${ }^{1}$.

A International Consensus Conference 2000 enfatiza a importância da MRPA, mas refere apenas que deve ser realizada após cinco minutos de repouso ${ }^{2}$. Pickering, em seu livro, exalta o valor da MRPA, mas não especifica quantas, quando e como realizar as medidas de MRPA ${ }^{3}$. Outras diretrizes e publicações, incluindo o VI JNC ${ }^{4}$, o VII $\mathrm{JNC}^{5}$, o WHO-ISH${ }^{6}$, o ESH-ESC ${ }^{7}$ e o First International Consensus Conference on Self-Blood Pressure Measurement ${ }^{8}$ não especificam as recomendações do procedimento. No entanto, todas as diretrizes recomendam medidas pela manhã e à noite na MRPA. Na American Society of Hypertension Ad Hoc Panel, Pickering recomenda a medida da PA pela MRPA em todos os dias (de trabalho ou não), mas não determina como deve ser feita 9 .

\section{Quantos dias o exame deve ser realizado?}

Com relação a quantos dias devem ser realizados os exames para obtenção da melhor média para a MRPA, observamos uma grande discrepância dos estudos na literatura. Alguns estudos obtêm os dados em um único dia ${ }^{10,11}$; outros obtiveram medidas em três ou quatro dias $^{12,13,14}$; alguns com pelo menos uma semana ${ }^{15,16,17,18,19}$ e até mais de um mês ${ }^{20}$. Há também grande divergência nos estudos analisados em relação ao número de medidas em cada dia: uma única medida pela manhã ${ }^{20}$, uma medida $^{10,11}$, duas ${ }^{14,18,19}$, ou três pela manhã e à noite ${ }^{12,13,15,16}$.

\section{Quantas medidas devem ser realizadas por dia?}

A reprodutibilidade da MRPA é diretamente proporcional ao número de medidas realizadas ${ }^{16,21}$. Foi demonstrado no estudo de Chatellier e cols. que a redução máxima no desvio-padrão das médias entre duas MRPA foi obtida com trinta medidas (dez dias consecutivos com três medidas por dia), e $80 \%$ da redução máxima foram conseguidos com cinco dias (quinze medidas) ${ }^{16}$.

0 primeiro estudo especificamente desenhado para determinar o número de medidas que deveriam ser realizadas na MRPA foi o de Stergiou e cols., realizado em 1998, com 189 hipertensos. Esse estudo demonstrou que as medidas do primeiro dia deveriam ser excluídas, e que os dois últimos dias são necessários para uma melhor reprodutibilidade, e superior à medida do consultório ${ }^{19}$. 0 mesmo autor confirmou posteriormente esses dados sugerindo ser necessário pelo menos três dias de monitorização (sempre excluindo o primeiro dia) ${ }^{18}$.

Em recentes diretrizes publicadas pela European Society of Hypertension Working Group on Blood Pressure Monitoring, sugere-se que os valores obtidos no primeiro dia de MRPA devem ser excluídos para o cálculo das médias ${ }^{22}$.

Celis e cols., estudando 74 pacientes idosos em dez dias de MRPA, concluíram que era necessária a realização de três dias de MRPA para se obter uma PA estável ${ }^{17}$. Brook reviu doze trabalhos com MRPA e, estudando a variabilidade das medidas da PA, o número total de medidas, o número total por série, o número de séries por dia e o número de dias de MRPA, concluiu que se pode obter um pequeno número de medidas e utilizar um protocolo simplificado de duas medidas em um dia ${ }^{23}$.

No estudo de Ohkubo e cols., realizado em população japonesa de Ohasama, com 1.491 pacientes com idade $\geq$ quarenta anos, acompanhados por um período médio de 10,6 anos, o valor preditivo foi maior quanto maior foi 
o número de medidas, chegando a elevar o risco de AVC em $35 \%$ com a elevação de $10 \mathrm{mmHg}$ na MRPA. Um dos dados mais interessantes é de que uma medida fora do consultório mostrou aumento significativo do risco de AVC quando comparado ao valor da PA convencional (média de duas medidas no consultório), com aumento de 19/8\% do risco de AVC por elevação inicial de 10 mmHg na MRPA/consultório da PA sistólica, respectivamente. Analisando esses dados, recomenda-se que preferencialmente se utilizem mais de 14 medidas. É importante enfatizar que a MRPA tem um maior valor preditivo para o risco de AVC que a medida convencional da $\mathrm{PA}$, mesmo em número reduzido de medidas ${ }^{20}$.

Chatellier e cols. sugerem quinze medidas na MRPA como o número recomendado, com a possibilidade de realizar mais medidas ${ }^{16}$. Já Stergiou e cols. propõem um número de doze medidas ${ }^{19}$. As diretrizes da European Society of Hypertension Working Group on Blood Pressure Monitoring recomendam duas medidas pela manhã e à noite, durante sete dias, excluindo-se o primeiro dia, com médias de 24 medidas (seis dias)².

\section{Conclusão}

Para se determinar o número ótimo de medidas para a realização da MRPA são necessárias informações adicionais, principalmente considerando as doenças cardiovasculares. A nosso ver, a MRPA deve seguir o seguinte protocolo: (a) realizado em dias úteis e representativos; (b) reservar um dia para entrega do equipamento e orientações ao paciente e/ou acompanhante; (c) quatro dias de monitorização; (d) duas séries por dia - manhã e noite (preferencialmente antes do desjejum e do jantar, ou após duas horas das refeições e antes das medicações); (e) três medidas por série (após três minutos de repouso e um minuto de intervalo entre elas); (f) excluir as medidas realizadas no primeiro dia de monitorização, por causa da reação de alarme; (g) total de dezoito medidas válidas para cálculo das médias; e (h) é aceitável número menor de medidas válidas, desde que não inferior a doze medidas e que todos os dias estejam representados.

\section{REFERÊNCIAS}

1. III Diretrizes para uso da Monitorização Ambulatorial da Pressão arterial. I Diretrizes para uso da Monitorização Residencial da Pressão Arterial. Rev Bras Hipertens 2001; 8: 143-55.

2. Asmar R, Zanchetti A. Guidelines for the use of self-blood pressure monitoring: a summary report of the first international consensus conference. J Hypertens 2000; 18: 493-508.

3. Pickering TG. Clinicians' Manual on Self-Monitoring of Blood Pressure. London: Science Press, 1997.

4. National High Blood Pressure Education Program: The sixth report of the Joint National Committee on Prevention, Detection, Evaluation, and Treatment of High Blood Pressure. Arch Intern Med 1997; 157: 2413-6.

5. Chobanian AV, Bakris GL, Black HR et al.: The National High Blood Pressure Education Program Coordinating Committee: the seventh report of the Joint National Committee on Prevention, Detection, Evaluation, and Treatment of High Blood Pressure: the JNC 7 report. JAMA 2003; 289: 2560-72.

6. Guidelines Subcommittee. 1999 World Health OrganizationInternational Society of Hypertension Guidelines for the Management of Hypertension. J Hypertens 1999; 17: 151-83.

7. Guideline Committee. 2003 European Society of HypertensionEuropean Society of Cardiology guidelines for the management of arterial hypertension. J Hypertens 2003; 21: 1011-53.

8. Mengden T, Chamontin B, Phong Chau N et al. User procedure for selfmeasurement of blood pressure. Blood Press Monit 2000; 5: 111-29.

9. Pickering T. Recommendation for the use of home (self) and ambulatory blood pressure monitoring. Am J Hypertens 1995; 9: 1-11.

10. Mancia G, Zanchetti A, Agabiti-Rosei E et al. for the SAMPLE Study Group. Ambulatory blood pressure is superior to clinic blood pressure in predicting treatment-induced regression of left ventricular hypertrophy. Circulation 1997; 95: 1464-70.

11. Mancia G, Sega R, Bravi C et al. Ambulatory blood pressure normality: results from the PAMELA study. J Hypertens 1995; 13: 1377-90.

12. Bodrie G, Genes N, Vaur L et al. Is "isolated home" hypertension as opposed to "isolated office" hypertension a sign of greater cardiovascular risk? Arch Intern Med 2001; 161: 2205-11.
13. Chatellier G, Dutrey-Dupagne C, Vaur L et al. Home self blood pressure measurement in general practice: the SMART study. Am J Hypertens 1996; 9: 644-52.

14. Stergiou GS, Thomopoulou GC, Skeva II, Mountokalakis TD. Home blood pressure normalcy: the Didima study. Am J Hypertens 2000; 13: 678-85.

15. Den Hond E, Celis H, Fagard R et al. on behalf of the THOP investigators. Self-measured versus ambulatory blood pressure in the diagnosis of hypertension. J Hypertens 2003; 21: 717-22.

16. Chatellier G, Day M, Bodrie G, Menard J. Feasibility study of N-of-1 trials with blood pressure self-monitoring in hypertension. Hypertension 1995; 25: 294-301.

17. Celis H, De Cort P, Fagard R, Thijs L, Staessen JA. For how many days should blood pressure be measured at home in older patients before steady levels are obtained? J Hum Hypertens 1997; 11: 673-7.

18. Stergiou GS, Baibas NM, Gantzarou AP et al. Reproducibility of clinic, ambulatory and home blood pressure: implications on the design of trials for the assessment of the efficacy of antihypertensive drugs. Am J Hypertens 2001 ; 15: 101-4.

19. Stergiou GS, Skeva II, Zourbaki AS, Mountokalakis TD. Self monitoring of blood pressure at home: how many measurements are needed? J Hypertens 1998; 16: 725-31.

20. Ohkubo T, Asayama K, Kikuya M et al. How many times should blood pressure be measured at home for better prediction of stroke risk? 10year follow-up results from the Ohasama study. J Hypertens 2004; 22:1099-104.

21. Imai Y, Ohkubo T, Hozawa A et al. Usefulness of home blood pressure measurements in assessing the effect of treatment in a single-blind placebo-controlled open trial. J Hypertens 2001; 19:179-85.

22. O'Brien E, Asmar R, Beilin L et al. on behalf of the European Society of Hypertension Working Group on Blood Pressure Monitoring. European Society of Hypertension recommendations for conventional, ambulatory and home blood pressure measurement. J Hypertens 2003; 21: 821-48.

23. Brook RD. Home blood pressure: accuracy is independent of monitoring schedules. Am J Hypertens 2000; 13: 625-31. 